\title{
Rotary Inertia and Higher Modes Effect on the Dynamic Response of Timoshenko Beams on Two-Parameter Elastic Foundation
}

\author{
Çağlayan HIZAL ${ }^{1}$ \\ Hikmet Hüseyin ÇATAL ${ }^{2}$
}

\begin{abstract}
This study investigates the effects of rotary inertia and higher modes on the dynamic response of the axially loaded Timoshenko beams on two-parameter foundation with generalized elastic end conditions. A simplified modal analysis procedure is presented by using the conventional separation of variables method. The effect of rotary inertia on the solution of free vibration differential equation is discussed. A numerical example is presented to investigate the coupled effect of rotary inertia and higher modes on the bending moment and shear force responses.
\end{abstract}

Keywords: Rotary inertia, higher modes effect, separation of variables method, two parameter-foundation.

\section{INTRODUCTION}

The dynamic behavior of axially loaded beams on elastic foundation has gained the attention of many researchers. The Winkler type foundation, which is the best known of the elastic soil models, has been widely used for the estimation of soil-structure interaction. A great number of studies exist in the literature regarding the dynamic response of Euler or Timoshenko beams on Winkler type foundations [1-2]. Çatal [3,4] obtained the fourth order differential equations by using the separation of variables method for the beams partially resting on Winkler foundation with the axial force and rotary inertia effects. A particular case, in which the free vibration equation of motion has five different solutions depending on the subgrade modulus was highlighted. Yeşilce and Çatal [5] investigated the free vibration of the Timoshenko beams on Winkler foundation with different subgrade reactions. Differential transform method (DTM) was adopted by Çatal [6] to the free vibration equations of the

\section{Note:}

- This paper has been received on March 23, 2018 and accepted for publication by the Editorial Board on November 12, 2018.

- Discussions on this paper will be accepted by September 30, 2019.

- https://dx.doi.org/10.18400/tekderg.408772

1 Izmir Institute of Technology, Dep. of Civil Engineering, İzmir, Turkey - caglayanhizal@iyte.edu.tr https://orcid.org/0000-0002-9783-6511

2 Dokuz Eylul University, Department of Civil Engineering, İzmir, Turkey - huseyin.catal@deu.edu.tr 
Timoshenko beams resting on Winkler type foundation and verified by the analytical results. Yeşilce et al. used the DTM for the free vibration analysis of axially loaded Reddy-Bickford beams [7-8]. Sapountzakis and Kampitsis [9] investigated the nonlinear behavior of the beams partially supported by tensionless Winkler foundation. Çatal [10] obtained the displacement response of forced Euler-Bernoulli beams on Winkler foundation by using the DTM. Öztürk and Coskun [11] obtained the analytical solution for the free vibration of beams on Winkler foundation with different support conditions.

In the Winkler model, the elastic soil is represented by independent linear springs within an infinitesimal part of the beam. More realistic approaches such as the two-parameter elastic foundation approach was proposed by Pasternak [12], and Vlasov and Leont'ev [13], respectively. These type of foundation models suppose that an interaction is constituted between Winkler springs by the transverse displacement [12-13]. This interaction is defined by a second parameter which represents the shear coefficient of an incompressible shear layer on the soil surface. In Pasternak model, the influence of the soil to both sides of the foundation beam is ignored as opposed to the Vlasov Model. Despite this difference, the second parameter can be taken equal for both models by considering the soil layer as a semi-infinite elastic medium [14]. Various researchers studied the behavior of the beams on two parameter foundations. Yokoyama [15] obtained the stiffness and mass matrices for the free vibration of Timoshenko beam-columns on two-parameter foundation with the effect of rotary inertia. Arboleda-Monsalve et al. [16] analyzed axially Timoshenko beam-columns with generalized end conditions on two parameter elastic foundation with rotary inertia by using the dynamic stiffness approach. Balkaya et al. [17] analyzed the dynamic response of the beams on Winkler and Pasternak foundation by using DTM. Celep et al. [18] calculated the response of a completely free beam on a tensionless Pasternak foundation subjected to dynamic loading. Malekzadeh and Karami [19] analyzed the free vibration of thick beams on twoparameter elastic foundations by using the differential quadrature and finite element method. Morfidis [20] obtained the exact finite element formulation for the dynamic analysis of beams on two and three-parameter foundations. Calio and Greco [21] investigated the exact free vibrations of Timoshenko beams and compared their results by Yokoyama [15]. Hassan and Nassar [22] obtained the dynamic displacement response of the axially loaded Timoshenko beams on two-parameter foundation.

Most previous studies investigate the dynamic response considering a few modes (commonly 3 or 5 modes). The effect of higher modes on the vibration is generally omitted. Although, the contribution of first 3-5 modes generally yield the exact results for displacement response, the bending moment and shear force response might be affected ultimately by the higher modes. Hizal and Çatal [23] mentioned that case and gave a small illustrative example. However, their study focused on the comparison of dynamic response of the Euler and Timoshenko beams on modified Vlasov foundation. In addition to the mentioned problems, the solution of the differential equation of free vibration might be affected by the rotary inertia in higher modes. For this reason, the required number of modes should be investigated considering the coupled effect of rotary inertia and higher modes.

In this study, a general solution for the dynamic behavior of axially loaded Timoshenko beams on two-parameter foundation with generalized end condition is presented. To the knowledge of the authors, the effect of higher modes on the dynamic response incorporated with the rotary inertia effect is not investigated in the literature. Different from the previous 
researches, a comprehensive study is presented to show the coupled effect of higher modes and rotary inertia on the dynamic response. The effect of the rotary inertia on the solution procedure is discussed. Modal variations in the bending moment and shear force responses of Euler and Timoshenko beams with respect to the foundation type, axial force and rotary inertia are presented.

\section{GOVERNING EQUATIONS}

In Fig. (1), the free body diagram of an infinitesimal part of an axially loaded Timoshenko beam element is presented. Here, the transverse force and moment equilibrium of the given infinitesimal part can be written as below according to the non-dimensional coordinate, $z=x / L$.

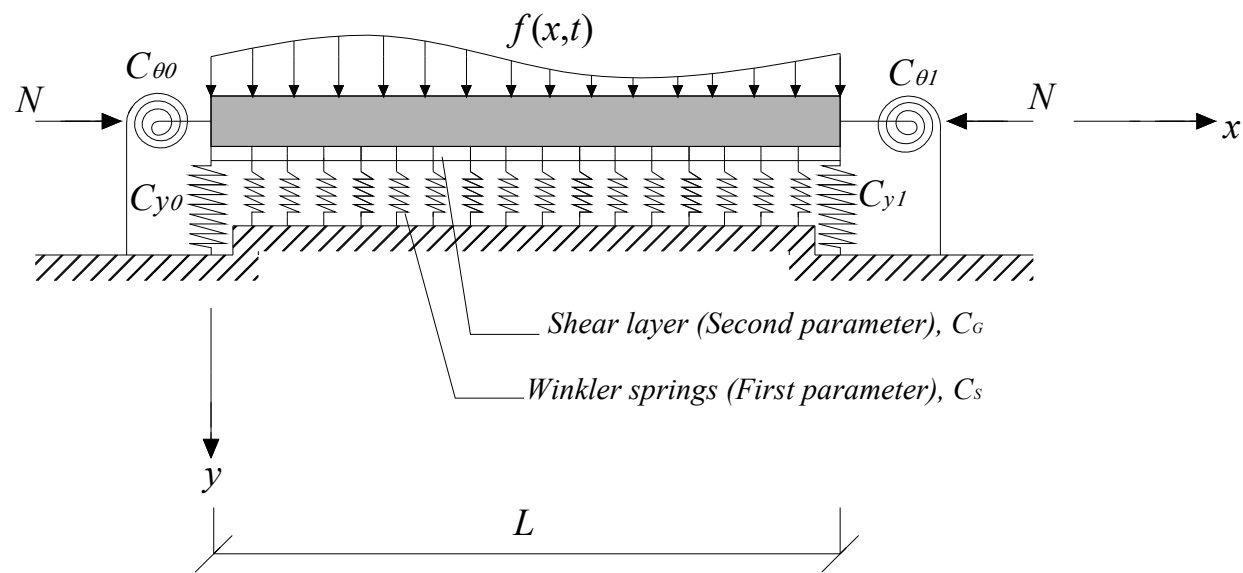

(a)

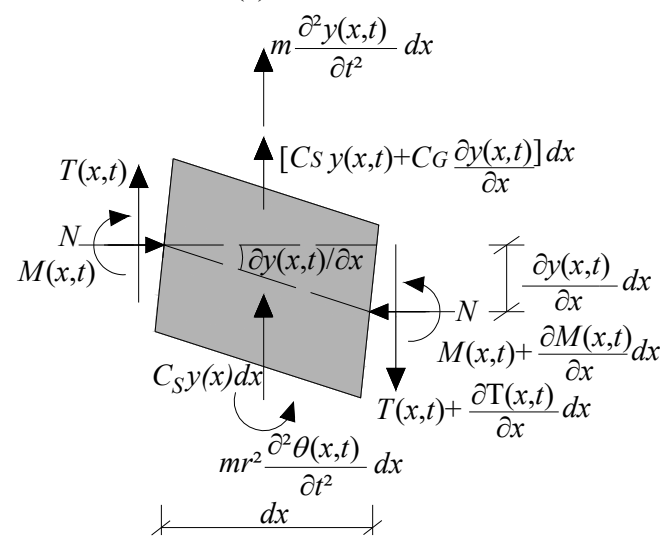

(b)

Figure 1 - (a) Timoshenko beam resting on two-parameter elastic foundation (b) Free body-diagram of infinitesimal element obtained from the beam 


$$
\begin{aligned}
& \frac{1}{L} \frac{\partial T(z, t)}{\partial z}=m \frac{\partial^{2} y(z, t)}{\partial t^{2}}+C_{S} y(z, t)-C_{G} \frac{1}{L^{2}} \frac{\partial^{2} y(z, t)}{\partial x^{2}}-f(z, t) \\
& \frac{1}{L} \frac{\partial M(z, t)}{\partial z}=\frac{1}{L} N \frac{\partial y(z, t)}{\partial z}-m r^{2} \frac{\partial^{2} \theta(z, t)}{\partial t^{2}}+T(z, t)
\end{aligned}
$$

In the equations above, $y(z, t), \theta(z, t), M(z, t), T(z, t)$, and $f(z, t)$ denote the transverse displacement, angle of rotation, bending moment and shear force functions, and the dynamic external load, respectively. Further, $N, m, r$ and $L$ are the axial compressive load, distributed mass, radius of gyration and length of the beam element. In addition, the coefficients of $C_{S}$ and $C_{G}$ represent the elastic spring coefficients of the soil. According to the Timoshenko beam theory, with the effect of axial deformation, the first and fourth order derivatives of transverse displacement with respect to $z$ are written as below [16];

$$
\begin{aligned}
& \left(1-\frac{N}{\kappa A G}\right) \frac{1}{L} \frac{\partial y(z, t)}{\partial z}=\theta(z, t)+\frac{T(z, t)}{\kappa A G} \\
& \left(1-\frac{N}{\kappa A G}\right) \frac{1}{L^{4}} \frac{\partial^{4} y(z, t)}{\partial z^{4}}=-\frac{1}{L^{2}} \frac{1}{E I} \frac{\partial^{2} M(z, t)}{\partial z^{2}}+\frac{1}{L^{3}} \frac{1}{\kappa A G} \frac{\partial^{3} T(z, t)}{\partial z^{3}}
\end{aligned}
$$

where $E, I, G, \kappa$ and $A$ denote the modulus of elasticity, second moment of inertia, shear modulus of the beam material, shear correction factor and area of the beam cross section, respectively. In addition, $E I$ and $\kappa A G$ denote the bending and shear rigidity of the beam cross section. Substituting Eq. (3) into Eq. (2) and taking the first order derivative with respect to $z$ leads to

$$
\frac{1}{L^{2}} \frac{\partial^{2} M(z, t)}{\partial z^{2}}=\frac{\partial T(z, t)}{\partial z}+\frac{1}{L^{2}} N \frac{\partial^{2} y(z, t)}{\partial z^{2}}-m r^{2}\left(\left[1-\frac{N}{\kappa A G}\right] \frac{1}{L^{2}} \frac{\partial^{4} y(z, t)}{\partial t^{2} \partial z^{2}}-\frac{1}{\kappa A G} \frac{\partial^{3} T(z, t)}{\partial t^{2} \partial z}\right)
$$

Substituting Eq. (5) into Eq. (4) gives

$$
\begin{aligned}
& {\left[1-\frac{N}{\kappa A G}\right] \frac{\partial^{4} y(z, t)}{\partial z^{4}}+\frac{L^{2}}{E I}\left[\frac{\partial T(z, t)}{\partial z}+\frac{1}{L^{2}} N \frac{\partial^{2} y(z, t)}{\partial z^{2}}-m r^{2}\left(\left[1-\frac{N}{\kappa A G}\right] \frac{1}{L^{2}} \frac{\partial^{4} y(z, t)}{\partial t^{2} \partial z^{2}}\right.\right.} \\
& \left.\left.-\frac{1}{\kappa A G} \frac{1}{L} \frac{\partial^{3} T(z, t)}{\partial t^{2} \partial z}\right)\right]-\frac{L}{\kappa A G} \frac{\partial^{3} T(z, t)}{\partial z^{3}}=0
\end{aligned}
$$

Finally, the equation of motion can be written as below by substituting Eq. (1) into (6), 


$$
\begin{aligned}
& {\left[1-\frac{N}{\kappa A G}\right] \frac{\partial^{4} y(z, t)}{\partial z^{4}}+\frac{1}{E I}\left[m L^{2} \frac{\partial^{2} y(z, t)}{\partial t^{2}}+C_{S} L^{2} y(z, t)-C_{G} \frac{\partial^{2} y(z, t)}{\partial z^{2}}-m r^{2}\left(1-\frac{N}{\kappa A G}\right) \frac{\partial^{4} y(z, t)}{\partial t^{2} \partial z^{2}}\right.} \\
& \left.+N \frac{\partial^{2} y(z, t)}{\partial z^{2}}\right]-\frac{1}{\kappa A G}\left[m L^{2} \frac{\partial^{4} y(z, t)}{\partial t^{2} \partial z^{2}}+C_{S} L^{2} \frac{\partial^{2} y(z, t)}{\partial z^{2}}-C_{G} \frac{\partial^{4} y(z, t)}{\partial z^{4}}\right]+\frac{m r^{2} L^{4}}{E I \kappa A G}\left[m \frac{\partial^{4} y(z, t)}{\partial t^{4}}\right. \\
& \left.+C_{S} \frac{\partial^{2} y(z, t)}{\partial t^{2}}-\frac{C_{G}}{L^{2}} \frac{\partial^{4} y(z, t)}{\partial z^{2} \partial t^{2}}\right]=\frac{L}{\kappa A G} \frac{\partial^{2} f(z, t)}{\partial z^{2}}+\frac{L^{2} f(z, t)}{E I}+\frac{L m r^{2}}{\kappa A G E I} \frac{\partial^{2} f(z, t)}{\partial t^{2}}
\end{aligned}
$$

\section{MODAL ANALYSIS PROCEDURE}

\subsection{Free Vibration Analysis}

In Eqs. (1-7), deformations and internal forces of the beam element are defined as depending on the location and time variables. These parameters can be divided into two parts with the implementation of the method of the separation of variables as follows;

$$
\begin{aligned}
& y(z, t)=\sum_{i=1}^{\infty} Y_{i}(z) q_{i}(t) \\
& \theta(z, t)=\sum_{i=1}^{\infty} \Theta_{i}(z) q_{i}(t) \\
& M(z, t)=\sum_{i=1}^{\infty} \bar{M}_{i}(z) q_{i}(t) \\
& T(z, t)=\sum_{i=1}^{\infty} \bar{T}_{i}(z) q_{i}(t)
\end{aligned}
$$

where $i$ denotes the mode number and $Y_{i}(z), \Theta_{i}(z), \bar{M}_{i}(z), \bar{T}_{i}(z)$ and $\mathrm{q}_{\mathrm{i}}(\mathrm{t})$ are the displacement, angle of rotation, bending moment, shear force shape functions and the normal coordinate function, respectively. In the free vibration case (for $f(z, t)=0$ ), the normal coordinate function can be taken as $q_{i}(t)=\sin \left(\omega_{i} t+\varphi\right)$. Thus, the Eq. (7) can be written in the following form.

$$
\begin{aligned}
& Y_{i}^{I V}(z)+L^{2}\left(\frac{\kappa A G}{\kappa A G-N+C_{G}}\right)\left[\frac{m \omega_{i}^{2}-C_{s}}{\kappa A G}+\frac{\left(m \omega_{i}^{2} r^{2}+N-C_{G}\right)}{E I}+\frac{m \omega_{i}{ }^{2} r^{2}\left(C_{G}-N\right)}{E I \kappa A G}\right] Y_{i}^{I I}(z) \\
& +L^{4}\left(\frac{\kappa A G}{\kappa A G-N+C_{G}}\right)\left[\frac{m r^{2}}{E I \kappa A G}\left(m \omega_{i}^{4}-C_{s} \omega_{i}{ }^{2}\right)-\frac{\left(m \omega_{i}^{2}-C_{s}\right)}{E I}\right] Y_{i}(z)=0
\end{aligned}
$$


where $\omega_{i}$ and $\varphi$ denote the natural angular frequency of $i^{\text {th }}$ mode and the phase angle. Then, the shape functions of the bending moment, shear force and angle of rotation are obtained as [23].

$$
\begin{aligned}
& \bar{M}_{i}(z)=E I\left[\left(\frac{C_{s}-m \omega_{i}^{2}}{\kappa A G}\right) Y_{i}(z)-\left(\frac{C_{G}-N}{\kappa A G}+1\right) \frac{Y_{i}^{l l}(z)}{L^{2}}\right] \\
& \bar{T}_{i}(\xi)=\frac{M_{i}^{l}(z)-\left(m \omega_{i}^{2} r^{2}+N\right) Y_{i}^{l}(z)}{\left(1-\frac{m \omega_{i}{ }^{2} r^{2}}{\kappa A G}\right) L} \\
& \Theta_{i}(z)=\left(1-\frac{N}{\kappa A G}\right) \frac{Y_{i}^{l}(z)}{L}-\frac{\bar{T}_{i}(z)}{\kappa A G}
\end{aligned}
$$

In Eq. (13), it is seen that the displacement shape function is required to obtain $\bar{M}_{i}(z)$, $\bar{T}_{i}(z)$ and $\Theta_{i}(z)$. For this reason, the displacement shape function should be obtained at first. For this purpose, Eq. (12) can be written as;

$$
Y_{i}^{I V}(z)+a_{i} Y_{i}^{I I}(z)+b_{i} Y_{i}(z)=0
$$

where

$$
\begin{aligned}
& a_{i}=\left(\frac{L^{2} \kappa A G}{\kappa A G+C_{G}}\right)\left[\frac{m \omega_{i}^{2}-C_{s}}{\kappa A G}+\frac{\left(m \omega_{i}^{2} r^{2}+N-C_{G}\right)}{E I}+\frac{m \omega_{i}^{2} r^{2}\left(C_{G}-N\right)}{E I \kappa A G}\right] \\
& b_{i}=\left(\frac{m r^{2} \omega_{i}^{2}}{\kappa A G}-1\right)\left[\frac{L^{4} \kappa A G\left(m \omega_{i}^{2}-C_{s}\right)}{\left(\kappa A G+C_{G}-N\right) E I}\right]
\end{aligned}
$$

The roots of the differential equation given in Eq. (14) are obtained as;

$$
\lambda_{1}^{2}=\frac{-a_{i}-\sqrt{a_{i}^{2}-4 b_{i}}}{2} \quad \lambda_{2}{ }^{2}=\frac{-a_{i}+\sqrt{a_{i}{ }^{2}-4 b_{i}}}{2}
$$

Although the differential equation given in Eq. (14) involves six different solutions depending on the sign of $\lambda_{1}{ }^{2}$ and $\lambda_{2}{ }^{2}$, only three of them, Case-I: $\lambda_{1}{ }^{2}<0$ and $\lambda_{2}{ }^{2}>0$, Case-II: $\lambda_{1}{ }^{2}<0$ and $\lambda_{2}{ }^{2}=0$, and Case-III: $\lambda_{1}{ }^{2}<0$ and $\lambda_{2}{ }^{2}<0$ are physically possible. If the beam rests on a uniform elastic soil and the rotary inertia is considered, the lower modes satisfy Case-I for $\omega_{i}^{2}<\kappa A G /\left(m r^{2}\right)$. However, this case is violated for higher modes and case-II and III is satisfied for $\omega_{i}^{2}=\kappa A G /\left(m r^{2}\right)$ and $\omega_{i}^{2}>\kappa A G /\left(m r^{2}\right)$, respectively. In the case that the rotary inertia is neglected, the Case-I will satisfy all modes since the $\kappa A G /\left(m r^{2}\right)$ will be infinite. Thus, the shape functions are obtained for Case-I, Case-II and Case-III as follows.

For Case-I: $\lambda_{1}{ }^{2}<0$ and $\lambda_{2}{ }^{2}>0$; 
$Y_{i}(z)=C_{1} \cos \lambda_{1} z+C_{2} \sin \lambda_{1} z+C_{3} \cosh \lambda_{2} z+C_{4} \sinh \lambda_{2} z$

$\Theta_{i}(z)=C_{1} K_{5} \sin \lambda_{1} z+C_{2} K_{6} \cos \lambda_{1} z+C_{3} K_{7} \sinh \lambda_{2} z+C_{4} K_{7} \cosh \lambda_{2} z$

$\bar{M}_{i}(z)=C_{1} K_{1} \cos \lambda_{1} z+C_{2} K_{1} \sin \lambda_{1} z+C_{3} K_{2} \cosh \lambda_{2} z+C_{4} K_{2} \sinh \lambda_{2} z$

$$
\bar{T}_{i}(z)=C_{1} K_{3} \sin \lambda_{1} z-C_{2} K_{3} \cos \lambda_{1} z+C_{3} K_{4} \sinh \lambda_{2} z+C_{4} K_{4} \cosh \lambda_{2} z
$$

where

$\lambda_{1}=\sqrt{\frac{a_{i}+\sqrt{a_{i}^{2}-4 b_{i}}}{2}} \quad \lambda_{2}=\sqrt{\frac{-a_{i}+\sqrt{a_{i}^{2}-4 b_{i}}}{2}}$

and

$$
\begin{aligned}
& K_{1}=\left(\frac{\mathrm{EI}\left(\mathrm{C}_{\mathrm{s}}-\mathrm{m} \omega_{i}^{2}\right)}{\kappa \mathrm{AG}}\right)+\left(\frac{C_{G}-N}{\kappa A G}+1\right) \frac{E I \lambda_{1}^{2}}{L^{2}}, \\
& K_{2}=\left(\frac{\mathrm{EI}\left(\mathrm{C}_{\mathrm{s}}-\mathrm{m} \omega_{i}^{2}\right)}{\kappa \mathrm{AG}}\right)-\left(\frac{C_{G}-N}{\kappa A G}+1\right) \frac{E I \lambda_{2}^{2}}{L^{2}} \\
& K_{3}=\frac{\lambda_{1}\left(-K_{1}+\left(\mathrm{m} \omega_{i}^{2} r^{2}+N\right)\right)}{\mathrm{L}\left(-\mathrm{m} \omega_{i}^{2} r^{2} / \mathrm{kAG}+1\right)}, K_{4}=\frac{\lambda_{2}\left(K_{2}-\left(\mathrm{m} \omega_{i}^{2} r^{2}+N\right)\right)}{\mathrm{L}\left(-\mathrm{m} \omega_{i}^{2} r^{2} / \mathrm{kAG}+1\right)}, K_{5}=\left(\frac{-\lambda_{1}}{\mathrm{~L}}-\frac{K_{3}}{\kappa \mathrm{AG}}\right) \\
& K_{6}=\frac{\lambda_{1}}{\mathrm{~L}}\left(1-\frac{N}{\kappa \mathrm{AG}}\right)-\frac{K_{3}}{\kappa \mathrm{AG}}, K_{7}=\frac{\lambda_{2}}{\mathrm{~L}}\left(1-\frac{N}{\kappa \mathrm{AG}}\right)-\frac{K_{4}}{\kappa \mathrm{AG}}
\end{aligned}
$$

For Case-II: $\lambda_{1}^{2}<0$ and $\lambda_{2}^{2}=0$;

$$
\begin{aligned}
& Y_{i}(z)=C_{1} \cos \lambda_{1} z+C_{2} \cos \lambda_{1} z+C_{3} z+C_{4} \\
& \Theta_{i}(z)=C_{1} K_{5} \sin \lambda_{1} z-C_{2} K_{6} \sin \lambda_{1} z+C_{3} K_{7} \\
& \bar{M}_{i}(z)=C_{1} K_{1} \cos \lambda_{1} z+C_{2} K_{1} \cos \lambda_{1} z+C_{3} K_{2} z+C_{4} K_{2} \\
& \bar{T}_{i}(z)=C_{1} K_{3} \sin \lambda_{1} z-C_{2} K_{3} \sin \lambda_{1} z+C_{3} K_{4}
\end{aligned}
$$

and 


$$
\begin{aligned}
& K_{1}=\left(\frac{\mathrm{EI}\left(\mathrm{C}_{\mathrm{s}}-\mathrm{m} \omega_{i}^{2}\right)}{\kappa \mathrm{AG}}\right)+\left(\frac{C_{G}-N}{\kappa A G}+1\right) \frac{E I \lambda_{1}^{2}}{L^{2}}, K_{2}=\left(\frac{\mathrm{EI}\left(\mathrm{C}_{\mathrm{s}}-\mathrm{m} \omega_{i}^{2}\right)}{\kappa \mathrm{AG}}\right) \\
& K_{3}=\frac{\lambda_{1}\left(-K_{1}+\left(\mathrm{m} \omega_{i}^{2} r^{2}+N\right)\right)}{\mathrm{L}\left(-\mathrm{m} \omega_{i}^{2} r^{2} / \mathrm{kAG}+1\right)}, K_{4}=\frac{\lambda_{2}\left(-K_{2}+\left(\mathrm{m} \omega_{i}^{2} r^{2}+N\right)\right)}{\mathrm{L}\left(-\mathrm{m} \omega_{i}^{2} r^{2} / \mathrm{kAG}+1\right)}, K_{5}=\left(-\frac{\lambda_{1}}{\mathrm{~L}}-\frac{K_{3}}{\kappa \mathrm{AG}}\right) \\
& K_{6}=\frac{\lambda_{1}}{\mathrm{~L}}\left(1-\frac{N}{\kappa \mathrm{AG}}\right)-\frac{K_{3}}{\kappa \mathrm{AG}}, K_{7}=-\frac{1}{\mathrm{~L}}\left(1-\frac{N}{\kappa \mathrm{AG}}\right)-\frac{K_{4}}{\kappa \mathrm{AG}}
\end{aligned}
$$

For Case-III: $\lambda_{1}{ }^{2}<0$ and $\lambda_{2}{ }^{2}<0$;

$$
\begin{aligned}
& Y_{i}(z)=C_{1} \cos \lambda_{1} z+C_{2} \sin \lambda_{1} z+C_{3} \cos \lambda_{2} z+C_{4} \sin \lambda_{2} z \\
& \Theta_{i}(z)=C_{1} K_{5} \sin \lambda_{1} z+C_{2} K_{6} \cos \lambda_{1} z+C_{3} K_{7} \sin \lambda_{2} z+C_{4} K_{8} \cos \lambda_{2} z \\
& \bar{M}_{i}(z)=C_{1} K_{1} \cos \lambda_{1} z+C_{2} K_{1} \sin \lambda_{1} z+C_{3} K_{2} \cos \lambda_{2} z+C_{4} K_{2} \sin \lambda_{2} z \\
& \bar{T}_{i}(z)=C_{1} K_{3} \sin \lambda_{1} z-C_{2} K_{3} \cos \lambda_{1} z+C_{3} K_{4} \sin \lambda_{2} z-C_{4} K_{4} \cos \lambda_{2} z
\end{aligned}
$$

where

$$
\lambda_{1}=\sqrt{\frac{a_{i}+\sqrt{a_{i}{ }^{2}-4 b_{i}}}{2}} \lambda_{2}=\sqrt{\frac{a_{i}-\sqrt{a_{i}{ }^{2}-4 b_{i}}}{2}}
$$

and

$$
\begin{aligned}
& K_{1}=\left(\frac{\mathrm{EI}\left(\mathrm{C}_{\mathrm{s}}-\mathrm{m} \omega_{i}^{2}\right)}{\kappa \mathrm{AG}}\right)+\left(\frac{C_{G}-N}{\kappa A G}+1\right) \frac{E I \lambda_{1}^{2}}{L^{2}}, K_{2}=\left(\frac{\mathrm{EI}\left(\mathrm{C}_{\mathrm{s}}-\mathrm{m} \omega_{i}^{2}\right)}{\kappa \mathrm{AG}}\right)+\left(\frac{C_{G}-N}{\kappa A G}+1\right) \frac{E I \lambda_{2}{ }^{2}}{L^{2}} \\
& K_{3}=\frac{\lambda_{1}\left(-K_{1}+\left(\mathrm{m} \omega_{i}^{2} r^{2}+N\right)\right)}{\mathrm{L}\left(-\mathrm{m} \omega_{i}^{2} r^{2} / \mathrm{kAG}+1\right)}, K_{4}=\frac{\lambda_{2}\left(-K_{2}+\left(\mathrm{m} \omega_{i}^{2} r^{2}+N\right)\right)}{\mathrm{L}\left(-\mathrm{m} \omega_{i}^{2} r^{2} / \mathrm{kAG}+1\right)}, K_{5}=\left(-\frac{\lambda_{1}}{\mathrm{~L}}-\frac{K_{3}}{\kappa \mathrm{AG}}\right) \\
& K_{6}=\frac{\lambda_{1}}{\mathrm{~L}}\left(1-\frac{N}{\kappa \mathrm{AG}}\right)-\frac{K_{3}}{\kappa \mathrm{AG}}, K_{7}=-\frac{\lambda_{2}}{\mathrm{~L}}\left(1-\frac{N}{\kappa \mathrm{AG}}\right)-\frac{K_{4}}{\kappa \mathrm{AG}}, K_{8}=\frac{\lambda_{2}}{\mathrm{~L}}\left(1-\frac{N}{\kappa \mathrm{AG}}\right)+\frac{K_{4}}{\kappa \mathrm{AG}}
\end{aligned}
$$

An iterative computer program that determines the natural angular frequencies and mode shapes for the beam on an elastic foundation has been prepared by the authors. This program obtains the natural angular frequencies, and mode shapes for generalized end conditions. Here, the end conditions are defined by elastic supports with the coefficients of $C_{y 0}, C_{\theta 0}, C_{y l}$, $C_{\theta 1}$ as below; 


$$
\begin{aligned}
& C_{y 0} y(z=0)+T(z=0)=0 \\
& C_{\theta 0} \theta(z=0)+M(z=0)=0 \\
& C_{y 1} y(z=1)+T(z=1)=0 \\
& C_{\theta 1}(z=1)+M(z=1)=0
\end{aligned}
$$

Then, the coefficient matrix is obtained as follows;

for Case-I;

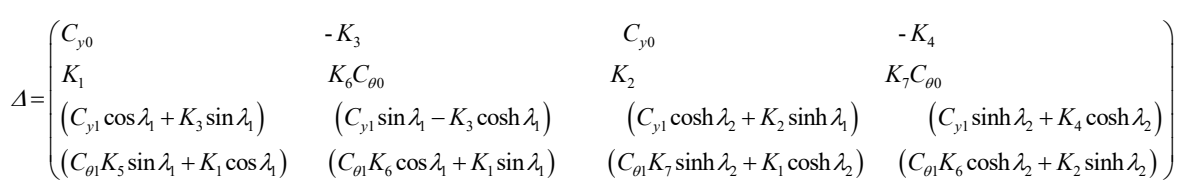

for Case-II;

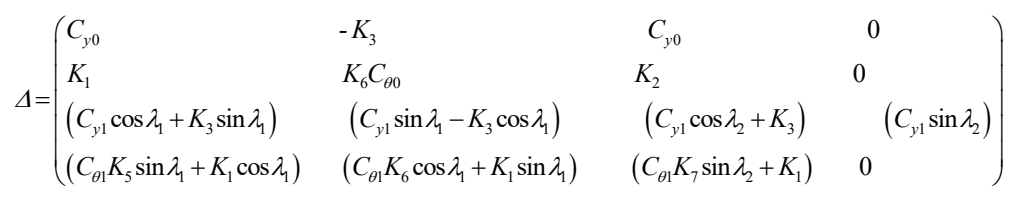

for Case-III;

$$
\Delta=\left(\begin{array}{llcr}
C_{y 0} & -K_{3} & C_{y 0} & -K_{4} \\
K_{1} & K_{6} C_{\theta 0} & K_{2} & K_{7} C_{\theta 0} \\
\left(C_{y 1} \cos \lambda_{1}+K_{3} \sin \lambda_{1}\right) & \left(C_{y 1} \sin \lambda_{1}-K_{3} \cos \lambda_{1}\right) & \left(C_{y 1} \cos \lambda_{2}+K_{3} \sin \lambda_{1}\right) & \left(C_{y 1} \sin \lambda_{2}-K_{4} \cos \lambda_{2}\right) \\
\left(C_{\theta 1} K_{5} \sin \lambda_{1}+K_{1} \cos \lambda_{1}\right) & \left(C_{\theta 1} K_{6} \cos \lambda_{1}+K_{1} \sin \lambda_{1}\right) & \left(C_{\theta 1} K_{7} \sin \lambda_{2}+K_{1} \cos \lambda_{21}\right) & \left(C_{\theta 1} K_{8} \cos \lambda_{2}+K_{2} \sin \lambda_{2}\right)
\end{array}\right)
$$

The coefficients of $C_{2}, C_{3}$ and $C_{4}$ can be obtained as below by setting, $C_{1}=1$.

$$
\left(\begin{array}{l}
C_{2} \\
C_{3} \\
C_{4}
\end{array}\right)=\left(\begin{array}{lll}
\Delta_{22} & \Delta_{23} & \Delta_{34} \\
\Delta_{32} & \Delta_{33} & \Delta_{34} \\
\Delta_{42} & \Delta_{43} & \Delta_{44}
\end{array}\right)^{-1} \times\left(\begin{array}{l}
-\Delta_{21} \\
-\Delta_{31} \\
-\Delta_{41}
\end{array}\right)
$$

Once, $C_{2}, C_{3}$ and $C_{4}$ are obtained, $Y_{i}(z)$ can be normalized so that its maximum value will be equal to 1 .

\subsection{Forced Vibration Analysis}

Once the natural angular frequencies and free vibration mode shapes are obtained, the rest of the problem can be easily solved numerically. The forced vibration case can be handled as a set of linear algebraic equations by deriving the uncoupled equations of motion. Finally, a 
linear dynamic analysis can be performed. For this propose, the separation of variables method can be applied to the Eqs. (1) and (2).

$$
\begin{aligned}
& \sum_{i=1}^{\infty}\left[m Y_{i}(\xi) \ddot{q}_{i}(t)+\left(C_{S} Y_{i}(z)-\frac{1}{L^{2}} C_{G} Y_{i}^{l l}(\xi)-\frac{1}{L} \bar{T}_{i}^{l}(z)\right) q_{i}(t)\right]=f(z, t) \\
& \sum_{i=1}^{\infty}\left[m r^{2} \Theta_{i}(z) \ddot{q}_{i}(t)+\left(\frac{1}{L} \bar{M}_{i}^{l}(z)-\frac{1}{L} N Y_{i}^{l}(z)-\bar{T}_{i}(z)\right) q_{i}(t)\right]=0
\end{aligned}
$$

Multiplying the both sides of Eq. (40) by $Y_{j}(z)$, and Eq. (41) by $\Theta_{j}(z)$, and integrating them along the beam length, the following equation is obtained according to the rule of the orthogonality of modes.

$$
\begin{aligned}
& \int_{0}^{1} m L Y_{j}^{2}(z) \ddot{q}_{j}(t) d z+\int_{0}^{1} Y_{j}(z)\left(L C_{S} Y_{j}(z)-\frac{C_{G} Y_{j}^{I I}(z)}{L}-\bar{T}_{j}(z)\right) q_{j}(t) d z=\int_{0}^{1} m L Y_{j}(z) \ddot{q}_{j}(t) d z \\
& \int_{0}^{1} m L \Theta_{j}^{2}(z) \ddot{q}_{j}(t) d z+\int_{0}^{1} \Theta_{j}(z)\left(\bar{M}^{I}(z)-N Y_{j}^{I}(z)-L \bar{T}_{j}(z)\right) q_{j}(t) d z=0
\end{aligned}
$$

Assembling Eqs. (42) and (43) yields,

$$
M_{j} \ddot{q}_{j}(t)+K_{j} q_{j}(t)=F_{j}(t)
$$

where $M_{j}, K_{j}$, and $F_{j}(\mathrm{t})$ denote the generalized mass, stiffness, and load at the $j^{\text {th }}$ mode, respectively.

$$
\begin{gathered}
M_{j}=m L \int_{0}^{1}\left[Y_{j}^{2}(z)+r^{2} \Theta_{j}^{2}(z)\right] d z \\
K_{j}=\int_{0}^{1}\left\{Y_{j}(z)\left(L C_{S} Y_{j}(z)-\frac{C_{G} Y_{j}^{I I}(z)}{L}-\bar{T}_{j}(z)\right)+\Theta_{j}(z)\left(\bar{M}^{I}(z)-N Y_{j}^{I}(z)-L \bar{T}_{j}(z)\right)\right\} d z \\
F_{j}(t)=L \int_{0}^{1} Y_{j}(z) f(z, t) d z
\end{gathered}
$$

Substituting $K_{j}=M_{j} \omega_{j}^{2}$ into Eq. (44) leads to the following equation

$$
\ddot{q}_{j}(t)+\omega_{j}^{2} q_{j}(t) d z=\frac{F_{j}(t)}{M_{j}}
$$

Thus, the normal coordinate function can be obtained by the solution of Eq. (46). 


\section{CALCULATION OF SOIL PARAMETERS}

The first parameter of the elastic soil which represents the modulus of transverse deformation can be evaluated by using the formulas given for the Winkler foundation model. On the other hand, the calculation of the second parameter is directly related to the type of the twoparameter elastic soil model. Vlasov and Leont'ev [13] proposed a formula for the calculation of the first and second parameters for rectangular beams on an elastic soil layer. These formulas were simplified by Zhaohua and Cook [24] for a semi-infinite elastic medium as given below.

$$
C_{S}=\frac{E_{o} \bar{b}}{2\left(1-v_{0}{ }^{2}\right)} \frac{\bar{\gamma}}{l} \quad C_{G}=\frac{E_{o} \bar{b}}{4\left(1+v_{0}\right)} \frac{l}{\bar{\gamma}}
$$

where, $\bar{b}$ denotes the width of the beam. The parameter of $\bar{\gamma}$ is defined by Vlasov and Leont'ev as a coefficient that characterizes the decrease of deflections with depth and commonly taken as $\bar{\gamma}=1$. Parameters of $l, E_{o}, v_{o}$ are given in the following equation.

$$
\begin{aligned}
& l=\sqrt[3]{\frac{2 E I\left(1-v_{0}^{2}\right)}{\left(1-v^{2}\right) E_{0} \bar{b}}} \\
& E_{o}=\frac{E_{s}}{1-v_{s}^{2}} \quad v_{o}=\frac{v_{s}}{1-v_{s}}
\end{aligned}
$$

where $E_{s}, v_{s}$ and $v$ denotes the modulus of elasticity and Poisson's ratio of the soil and the beam, respectively.

\section{NUMERICAL RESULTS AND DISCUSSIONS}

\subsection{Verification Example}

A numerical example which was previously studied by Yokoyama [15], and Calio and Greco [21] is presented to verify the presented solution procedure. The free vibration of the axially loaded hinged-hinged and fixed-hinged beam on Winkler and Pasternak foundation is investigated and analysis results are compared with those of the Yokoyama [15], and Calio and Greco [21]. The beam and soil properties are calculated according to the non-dimensional parameters below [25].

$$
\begin{aligned}
& \bar{n}=\frac{N L^{2}}{\pi^{2} E I} \\
& \bar{c}_{s}=\frac{C_{S} L^{2}}{E I}
\end{aligned}
$$


$\bar{c}_{g}=\frac{C_{G} L^{2}}{\pi^{2} E I}$

Where $\bar{n}, \bar{c}_{s}$ and $\bar{c}_{g}$ denotes the dimensionless axial force, the first and second parameters of the elastic soil. The dimensionless frequency parameters, $\Omega_{i}=E I /\left(L^{4} \omega_{\mathrm{i}}{ }^{2} m\right)$ of the first three modes obtained for the hinged-hinged and fixed-hinged beam are presented in Table- 1 and 2 , respectively. In Table-1, the frequency parameter obtained by the present study is found to be very close to the results presented by Yokoyama [15]. A small difference is obtained in second and third mode due to the fact that Yokoyama [15] presents a finite element solution by using 16 bar elements. Note that the difference with the Yokoyama solution [15] decreases for Vlasov type foundation as given in Table-2. In addition, a good agreement is obtained with the exact analytical results presented by Calio and Greco [21].

Table 1 - Frequency parameters obtained for $\bar{n}=0.6, \bar{c}_{s}=0.6 \pi^{4}$ and $\bar{c}_{g}=0(\kappa=2 / 3$ and $L / r=10.0)$

\begin{tabular}{ccccc}
\hline \multirow{2}{*}{ Mode Number } & \multicolumn{2}{c}{ Hinged-Hinged } & \multicolumn{2}{c}{ Fixed-Hinged } \\
\cline { 2 - 5 } & $\begin{array}{c}\text { Present } \\
\text { Study }\end{array}$ & $\begin{array}{c}\text { Yokoyama } \\
{[15]}\end{array}$ & $\begin{array}{c}\text { Present } \\
\text { Study }\end{array}$ & Yokoyama [15] \\
\hline 1 & 8.22 & 8.22 & 10.46 & 10.49 \\
2 & 20.59 & 20.67 & 22.20 & 22.30 \\
3 & 35.86 & 36.25 & 36.50 & 36.90 \\
\hline
\end{tabular}

Table 2 - Frequency parameters obtained for $\bar{n}=0.6, \bar{c}_{s}=0.6 \pi^{4}$ and $\bar{c}_{g}=1(\kappa=2 / 3$ and $L / r=10.0)$

\begin{tabular}{cccccc}
\hline \hline & \multicolumn{3}{c}{ Hinged-Hinged } & \multicolumn{2}{c}{ Fixed-Hinged } \\
\cline { 2 - 6 } \begin{tabular}{c} 
Mode \\
\cline { 2 - 6 }
\end{tabular} & $\begin{array}{c}\text { Present } \\
\text { Study }\end{array}$ & $\begin{array}{c}\text { Yokoyama } \\
{[15]}\end{array}$ & $\begin{array}{c}\text { Calio and } \\
\text { Greco [21] }\end{array}$ & Present Study & Yokoyama [15] \\
\hline 1 & 12.64 & 12.64 & 12.64 & 14.42 & 14.42 \\
2 & 28.03 & 28.10 & 28.02 & 29.30 & 29.34 \\
3 & 45.92 & 46.34 & 45.92 & 46.70 & 46.74 \\
\hline
\end{tabular}

The variation of dimensionless eigenvalues $\mu_{1}=\lambda_{1}^{2} E I /\left(L^{4} \omega_{\mathrm{i}}^{2} m\right)$ and $\mu_{2}=\lambda_{2}{ }^{2} E I /\left(L^{4} \omega_{\mathrm{i}}^{2} m\right)$ for the hinged-hinged and fixed-hinged support conditions are given in Figures 3-4, respectively. When the rotary inertia is neglected, Case-I is satisfied for all modes. For the hinged-hinged beam, when the rotary inertia is considered, Case-I is violated starting from the $4^{\text {th }}$ and $5^{\text {th }}$ modes, for the Winkler and Vlasov foundations, respectively. However, the higher modes satisfy Case-III. For the fixed-hinged beam, Case-I is satisfied up to the $3^{\text {rd }}$ mode. 


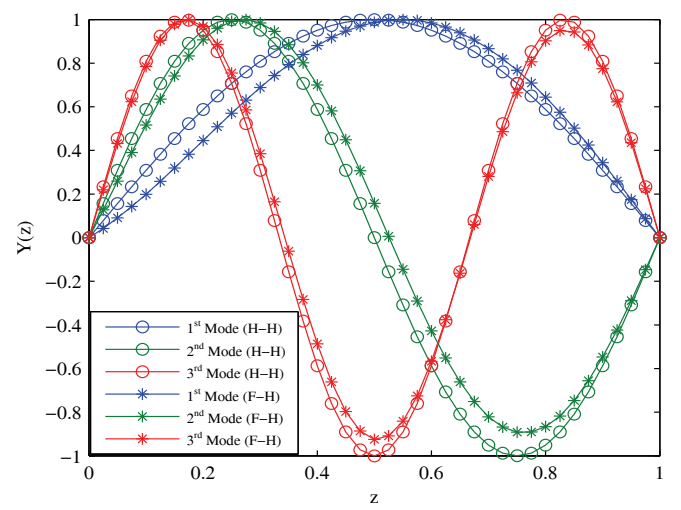

Figure 2 - Normalized mode shape functions obtained for first three modes
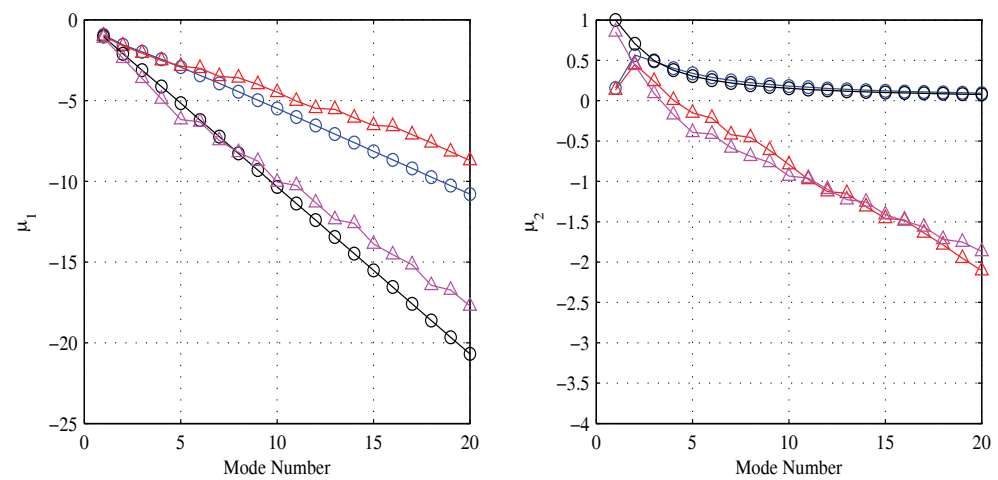

$\longrightarrow$ Winkler without R.I. $\quad \triangle \quad$ Winkler with R.I.

$\longrightarrow$ Vlasov without R.I. $\quad \triangle \quad$ Vlasov with R.I.

Figure 3 - Normalized eigenvalues for the hinged-hinged beam
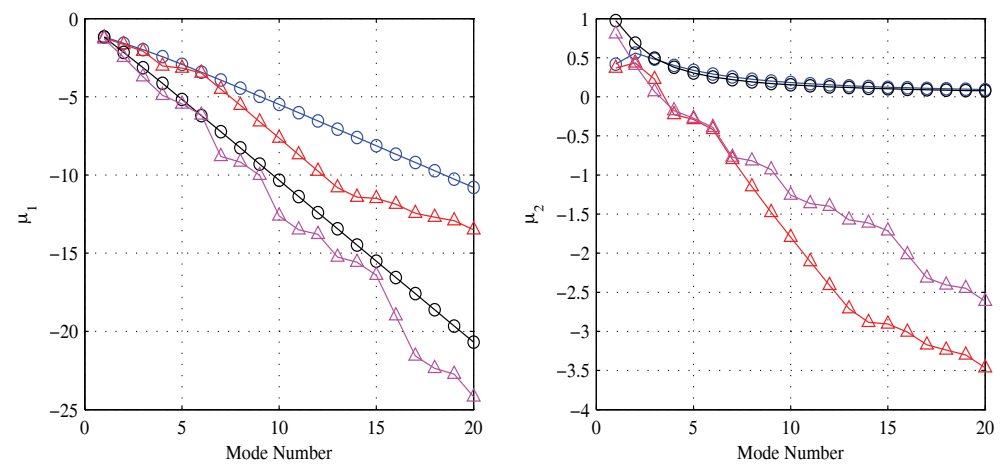

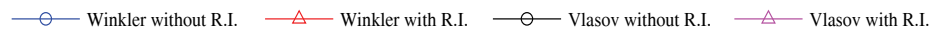

Figure 4 - Normalized eigenvalues for the fixed-hinged beam 


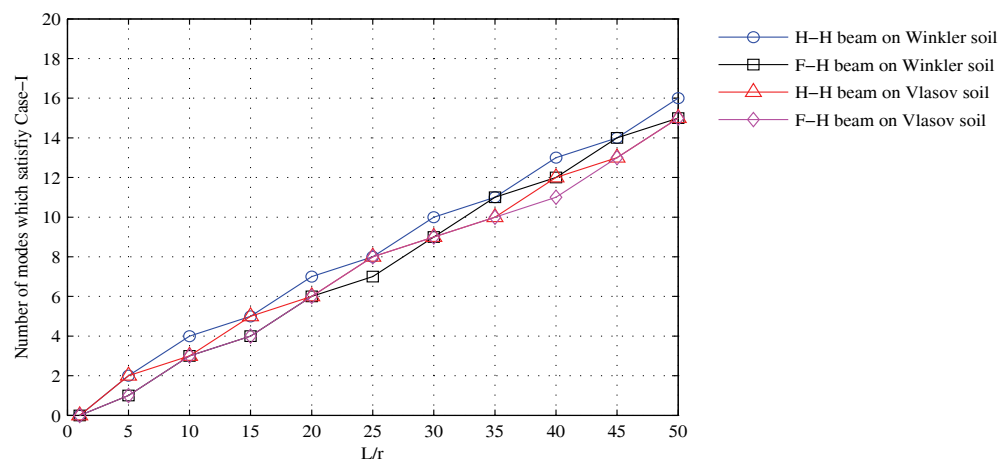

Figure 5 - Variation of the number of modes satisfying Case-I versus slenderness ratio

Figure 5 shows the variation of the number of modes that satisfy Case-I versus the slenderness ratio, $L / r$. It is seen that the number of modes that satisfy the Case-I is proportional to the slenderness ratio while some differences are observed according to the boundary conditions and soil model.

\subsection{Comprehensive Example}

In this numerical analysis, the dynamic response of an axially loaded $1^{m} \times 1^{m}$ square beam on Vlasov foundation is investigated. The beam has distributed mass and elasticity, and it is subjected to a concentrated dynamic load $f(z, t)=\delta(z-1 / 2) F(t)$. The material properties of the beam: $E=28,000 \mathrm{MPa}, G=11,666.67 \mathrm{MPa}, m=2.548 \mathrm{kN} . \mathrm{s}^{2} / \mathrm{m}, r=0.2887 \mathrm{~m}$ and $\kappa=2 / 3$. Elastic soil properties are calculated as $C_{S}=17,470 \mathrm{kN} / \mathrm{m}^{2}$ and $\mathrm{C}_{G}=68,688 \mathrm{kN}$ (for sand and gravel, $\left.E_{S}=100,000 \mathrm{MPa}, v_{\mathrm{s}}=0.25\right)$. In the analysis, four different boundary conditions; free-free $\left(C_{y 0}=C_{\theta 0}=C_{y l}=C_{\theta l}=0\right)$, hinged-hinged $\left(C_{y 0}=C_{y l} \approx \infty\right.$ and $\left.C_{\theta l}=C_{\theta 0}=0\right)$, fixedhinged $\left(C_{y 0}=C_{\theta 0}=C_{y l} \approx \infty\right.$ and $\left.C_{\theta I}=0\right)$, and fixed-fixed $\left(C_{y 0}=C_{\theta 0}=C_{y l}=C_{\theta l} \approx \infty\right)$ are considered. Axial compressive load of the beam is calculated by Euler critical buckling load formula, $N_{b}=\pi^{2} E I / L_{b}^{2}$, for all boundary conditions (see Table-3).
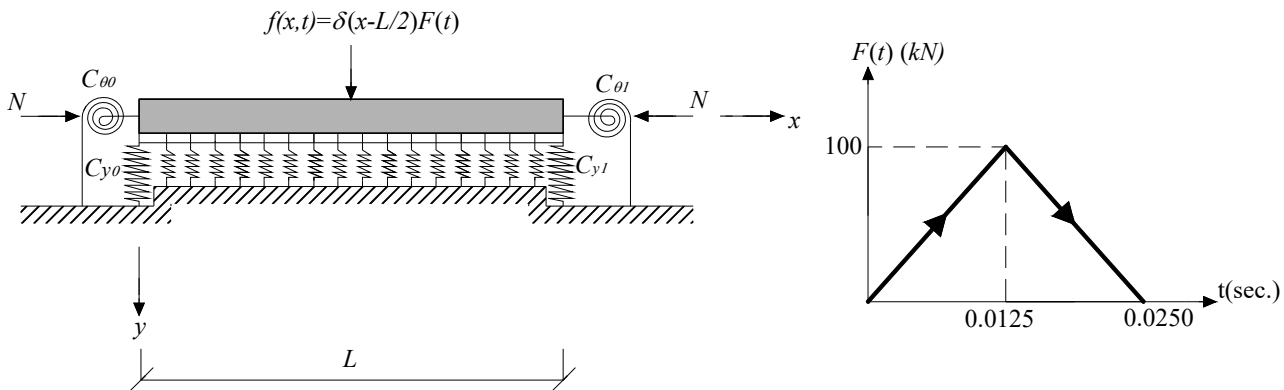

Figure 6 - Timoshenko beam on Vlasov foundation and time dependent load function 
Table 3 - Effective length and critical buckling loads according to the end conditions

\begin{tabular}{ccc}
\hline End Conditions & Effective Length, $L_{b}$ & Buckling Load, $N_{b}(k N)$ \\
\hline Free-Free & $L$ & 23029 \\
Hinged-Hinged & $L$ & 23029 \\
Fixed-Hinged & $0.7 L$ & 46998 \\
Fixed-Fixed & $0.5 L$ & 92116 \\
\hline
\end{tabular}

In Figures $7-8$, the variation of the maximum bending moment at $\mathrm{z} \approx 0.5$ versus the number of considered modes is presented. $95 \%$ convergence to the exact value is observed by the contribution of first 5-6 for all boundary conditions. In addition, it is observed that the maximum bending moment is less affected by the rotary inertia when the axial compressive force effect is omitted. An increase about 4\%, however, is observed for hinged-hinged beam on Winkler foundation with the rotary inertia effect. In addition, the bending moment response for the Winkler foundation is observed to be larger than Vlasov type foundation about 15, 8 and $7.5 \%$ for hinged-hinged, fixed-hinged and fixed-fixed cases, respectively. In the case where the axial compressive load is considered, the convergence speed of the bending moment response does not change significantly except for the free-free and hingedhinged beam on the Vlasov foundation. For this case, the exact response can be obtained by the first mode only. However, an increase 125, 312, 324\% in the bending moment response is observed for the free-free, hinged-hinged and fixed-hinged beam on Vlasov and Winkler foundations, while no difference is detected for the fixed-fixed beam.
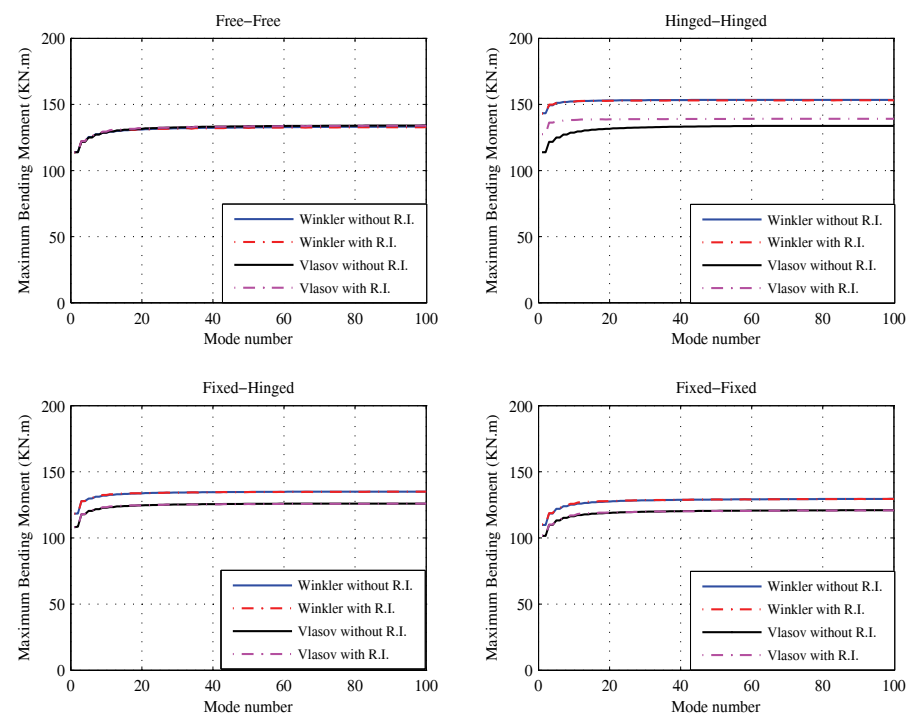

Figure 7 - Variation of maximum bending moment at $z=0.5$ versus considered number of modes for $N=0$ 

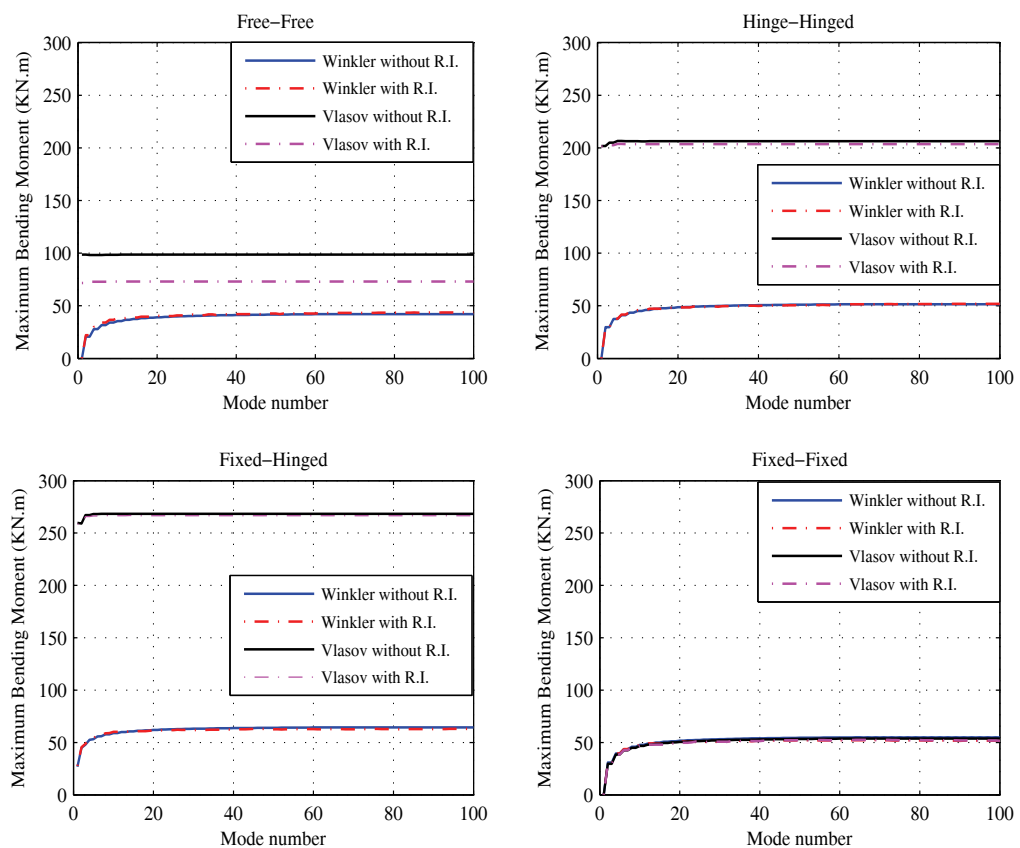

Figure 8 - Variation of maximum bending moment at $z=0.5$ versus considered number of modes for $N=N_{b}$

In Figures 9-10, the variation of the maximum shear force at $\mathrm{z} \approx 0.5$ versus considered mode number is presented. At first, it appears that the shear force responses with and without the rotary inertia are the same in lower modes for both Winkler and Vlasov foundations. However, a difference is observed with the contribution of higher modes. The exact shear force responses have similar values for Winkler and Vlasov foundations. The convergence to the exact value is obtained with less number of modes without the effect of rotary inertia. For the free-free and hinged-hinged beam, it is observed that taking of first 25-30 modes into account gives $90-95 \%$ convergence to the exact value without the rotary inertia effect. This convergence value, however, is satisfied by taking 40-45 modes into account if the rotary inertia is considered. For the fixed-hinged and fixed-fixed beam, this convergence is obtained by $15-20$ and 25-30 modes without and with the rotary inertia effect, respectively. On the other hand, in the case that the axial compressive load is considered, these convergence ratios are obtained for free-free and hinged-hinged beam without rotary inertia by the contribution of 35-40 modes and 55-60 modes with rotary inertia. For the fixed-hinged and fixed-fixed beam, $90-95 \%$ convergence is obtained by the contribution of 40-50 modes except the fixedfixed beam on Vlasov type foundation. If the axial compressive load is neglected, a small decrease occurs in the maximum shear force response for Winkler and Vlasov type foundations with rotary inertia. 

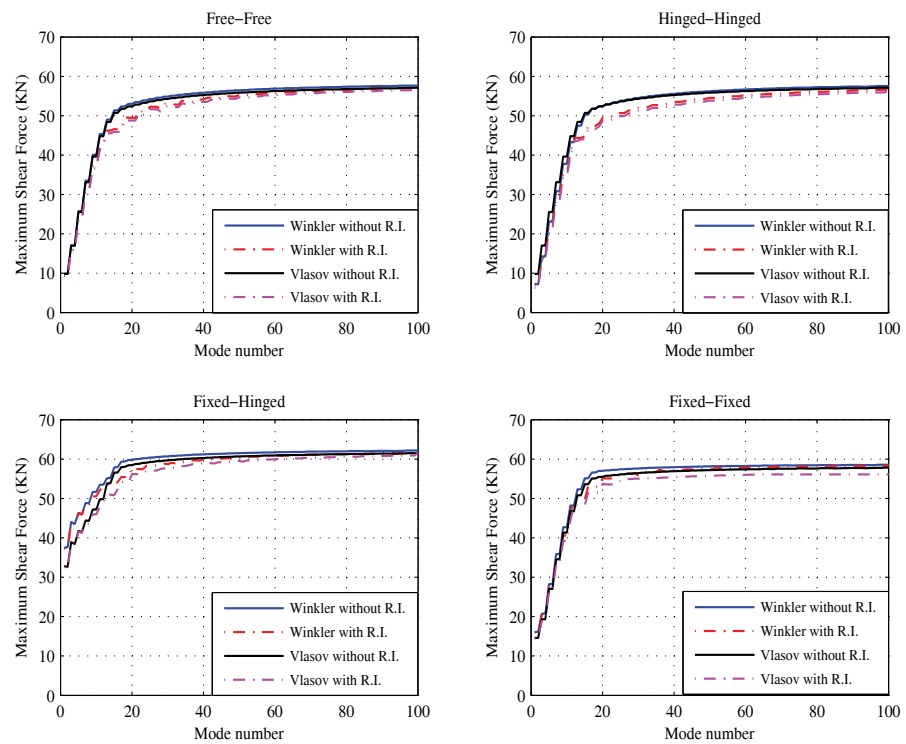

Figure 9 - Variation of maximum shear force at $z \approx 0.5$ versus considered number of modes for $N=0$
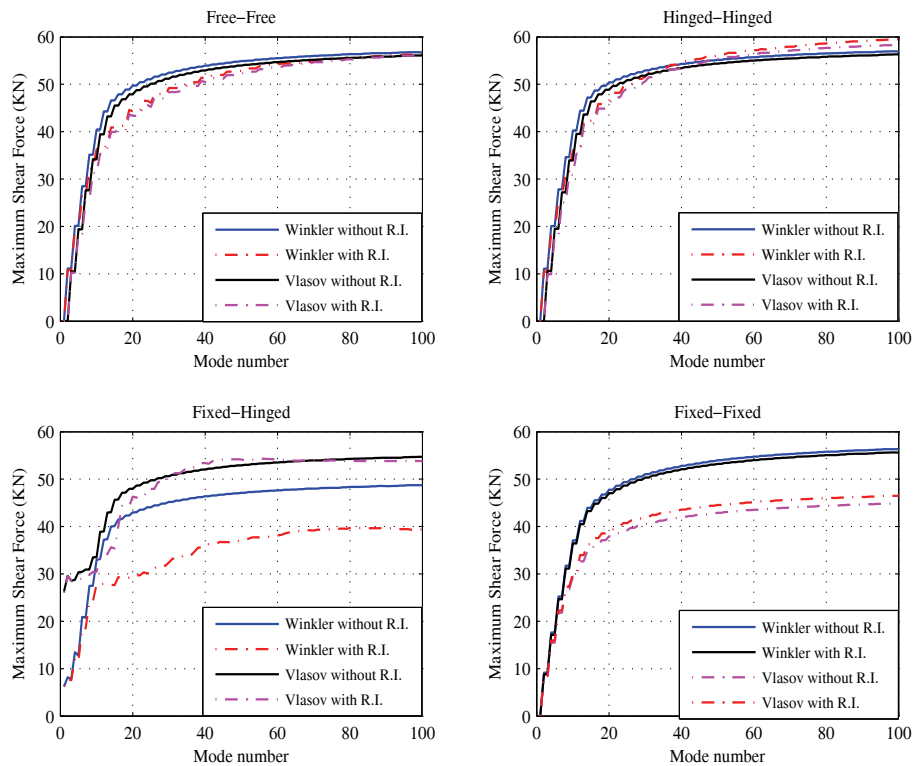

Figure 10 - Variation of maximum shear force at $z \approx 0.5$ versus considered number of modes for $N=N_{b}$ 
In the case when the axial compressive load contribution is considered, an increase about $4.50 \%, 20.50 \%$ for Winkler, and 3.50\%, 28\% for Vlasov foundation is observed by the effect of rotary inertia for the hinged-hinged and fixed-fixed beams, respectively. In addition, a decrease is observed about $19.25 \%$ for the fixed-hinged beam on Winkler foundation. Vlasov type foundation causes an increase about $11 \%$ and $38 \%$ in the shear force response for fixedhinged beam with and without the rotary inertia, respectively. On the other hand, a negligible difference is observed for free-free, hinged-hinged and fixed-fixed beams.

\section{CONCLUSIONS}

In this study, a comprehensive analysis on the effect of rotary inertia and higher modes on the dynamic response of Timoshenko beams on two-parameter foundation with generalized end condition is presented. The results are summarized below.

- In case of the consideration of rotary inertia, the solution of the differential equation for the free vibration case may change depending on the slenderness ratio, L/r. Here, three different cases (Case- I, II and III) are possible due to the slenderness ratio. In most researches in the literature, only the Case-I is considered as the solution. When the higher modes are considered, however, Case-II and/or III may arise.

- According to the numerical results, it is seen that the number of modes which satisfy Case-I increases with the slenderness ratio. In addition, it is seen that the number of modes that satisfy Case-I not only depends on the slenderness ratio but are also affected by the boundary conditions and elastic soil model.

- Numerical results indicate that the required number of modes increases by the effect of rotary inertia. On the other hand, the exact solution may be obtained by the contribution of less number of modes as depending on the number of constrained degrees of freedom at the ends.

- For the fixed-hinged beam on Winkler foundation and fixed-fixed beam on Winkler and Vlasov foundations, the axial compressive load causes a significant difference in the shear force response of the beam by the rotary inertia. In addition, the axial force increases the required number of modes for the shear force response except the fixedfixed beam with rotary inertia.

\section{References}

[1] Doyle, P.F. and Pavlovic, M., Vibration of beams on partial elastic foundations. Earthquake Engineering and Structural Dynamics, 10(5), 663-674, 1982.

[2] West, H. H. and Mafi, M., Eigenvalues for beam-columns on elastic supports. Journal of Structural Engineering, 110(6), 1305-1320, 1984.

[3] Çatal, H. H., Free vibration of partially supported piles with the effects of bending moment, axial and shear force. Engineering Structures, 24(12), 1615-1622, 2002. 
[4] Çatal, H. H., Free vibration of semi-rigid connected and partially embedded piles with the effects of the bending moment, axial and shear force. Engineering Structures, 28(14), 1911-1918, 2006.

[5] Yeşilce, Y. and Çatal, H. H., Free vibration of piles embedded in soil having different modulus of subgrade reaction. Applied Mathematical Modelling, 32(5), 889-900.

[6] Çatal, S., Solution of free vibration equations of beam on elastic soil by using differential transform method. Applied Mathematical Modelling, 32(9), 1744-1757, 2008.

[7] Yeşilce, Y. and Çatal, S., Free vibration of axially loaded Reddy-Bickford beam on elastic soil using the differential transform method, Struct. Eng. Mech. 31(4), 453-475, 2009.

[8] Yeşilce, Y. and Çatal, H. H., Solution of free vibration equations of semi-rigid connected Reddy-Bickford beams resting on elastic soil using the differential transform method. Archive of Applied Mechanics, 81(2), 199-213, 2011.

[9] Sapountzakis, E. J. and Kampitsis, A. E., Nonlinear dynamic analysis of Timoshenko beam-columns partially supported on tensionless Winkler foundation, Computers and Structures, 88(21-22), 1206-1219, 2010.

[10] Çatal, S., Response of forced Euler-Bernoulli beams using differential transform method. Struct. Eng. Mech. 42(1), 95-119, 2012.

[11] Özturk, B. and Coşkun, S. B., Analytical solution for free vibration analysis of beam on elastic foundation with different support conditions. Mathematical Problems in Engineering, 2013, 1-7, 2013.

[12] Pasternak P.L., On a new method of analysis on elastic foundation by means of two constants. Gosudarstvennoe Izdatel'stvo Literaturi po Stroitel'stvu i Arkhitekture, 1954.

[13] Vlasov, V.Z. and Leont'ev, U.N., Beams, plates and shells on elastic foundations. Israel Program for Scientific Translations, Jerusalem, 1966.

[14] Morfidis, K. and Avramidis, I. E., Formulation of a generalized beam element on a twoparameter elastic foundation with semi-rigid connections and rigid offsets. Computers and Structures, 80(25), 1919-1934, 2002.

[15] Yokoyama, T., Vibration analysis of Timoshenko beam-columns on two-parameter elastic foundation. Computers and Structures, 61(6), 995-1007, 1996.

[16] Arboleda-Monsalve, L. G., Zapata-Medina, D. G. and Aristizabal-Ochoa, J. D., Timoshenko beam-column with generalized end conditions on elastic foundation. Dynamic-stiffness matrix and load vector. Journal of Sound and Vibration, 310(4-5), 1057-1079, 2008.

[17] Balkaya, M., Kaya, M. O. and Sağlamer, A., Analysis of the vibration of an elastic beam supported on elastic soil using the differential transform method. Arch. Appl. Mech. 79(2), 135-146, 2009. 
[18] Celep, Z., Güler, K. and Demir, F., Response of a completely free beam on a tensionless Pasternak foundation subjected to dynamic load. Structural Engineering and Mechanics, 37(1), 61-77, 2011.

[19] Malekzadeh, P. and Karami, G., A mixed differential quadrature and finite element free vibration and buckling analysis of thick beams on two-parameter elastic foundations. Applied Mathematical Modelling 32(7), 1381-1394, 2008.

[20] Morfidis, K., Vibration of Timoshenko beams on three-parameter elastic foundation. Computers and Structures, 88(5-6), 294-308, 2010.

[21] Calio, I. and Greco, A., Free vibrations of Timoshenko beam-columns on Pasternak foundations. Journal of Vibration and Control. 19(5), 686-696, 2012.

[22] Hassan, M. T. and Nassar, M., Analysis of stressed Timoshenko beams on two parameter foundations. KSCE Journal of Civil Engineering, 19(1), 173-179, 2015.

[23] Hızal, Ç. and Çatal, H. H., Comparative dynamic analysis of axially loaded beams on modified Vlasov foundation. Structural Engineering and Mechanics, 57(6), 969-988, 2016.

[24] Zhaohua, F. and Cook, R.D., Beam elements on two-parameter elastic foundations. Journal of Engineering Mechanics, 109(6), 1390-402 (1983)

[25] De Rosa, M. A., Free Vibrations of Timoshenko Beams on Two Parameter Elastic Foundation. Computers and Structures, 57(1), 151-156, 1995. 